\title{
Viewpoint \\ Breast cancer metastasis: a microRNA story
}

Massimo Negrini ${ }^{1}$ and George Adrian Calin ${ }^{2}$

\begin{abstract}
${ }^{1}$ Department of Experimental and Diagnostic Medicine, Interdepartment Center for Cancer Research, University of Ferrara, via Luigi Borsari, Ferrara 44100, Italy

2Department of Experimental Therapeutics, University of Texas, MD Anderson Cancer Center, 1515 Holcombe Blvd, Houston, Texas 77030, USA
\end{abstract}

Corresponding author: George A Calin, gcalin@mdanderson.org

Published: 26 March 2008

This article is online at http://breast-cancer-research.com/content/10/2/303

(c) 2008 BioMed Central Ltd
Breast Cancer Research 2008, 10:303 (doi:10.1186/bcr1867)

A growing list of reports demonstrate that miRNAs play a critical role in cancer initiation and progression, and that miRNA alterations are ubiquitous among human cancers. Consequently, events that activate or inactivate miRNAs were regarded to cooperate with PCG abnormalities in human tumorigenesis [3]. For example, it was found that miRNAs contribute to oncogenesis by functioning as downregulated tumor suppressors, as is the case for the let-7 family, which target RAS oncogenes in lung cancers, and miR-15a and miR16-1, which target the BCL2 oncogene in chronic lymphocytic leukemias. It was also found that miRNAs can function as overexpressed oncogenes, as is the case for the miR17-92 cluster, which targetes the E2F1 oncogene in lymphomas, or miR-21, which regulates the PTEN tumor suppressor in hepatocellular carcinomas [4]. Much less was known about the upstream regulation of miRNA in cancer cells until recently, when a series of concomitant publications showed that the TP53 tumor suppressor regulates transcription of the miR-34 family (for review [5]) and mediate induction of apoptosis, cell cycle arrest, and senescence by TP53. Furthermore, it was shown that widespread miRNA repression by Myc contributes to tumorigenesis [6] in general and to repression of the oncogenic miR17-92 cluster [7] in particular.

\section{miRNAs as cancer players: a balance between repression of miRNA targets and regulation of miRNA expression}

MicroRNAs (miRNAs), which are short (19 to 24 nucleotides) noncoding genes that are excised from 60- to 110-nucleotide hairpin RNA precursors involved in the regulation of expression of protein-coding genes (PCGs), became a focal point in the molecular dissection of human cancer just a few few years ago [1]. They represent a new class of gene products whose functions are generally unknown. miRNA binds to target mRNA by imperfect complementarity, causing either mRNA degradation or translation inhibition. Recently, a deviation from the above perspective on miRNA function was identified; miR-369-3 can upregulate translation of tumor necrosis factor- $\alpha$ [2], which suggests an additional level of complexity in miRNA function.

\section{Breast cancer metastasis: a balance between suppression and activation by miRNAs}

Involvement of miRNA in tumor initiation and progression has come under intense scrutiny in recent years. The involvement of miRNAs in the development of metastases was initially discovered by $\mathrm{Ma}$ and coworkers [8] from Robert Weinberg's group who found that miR-10b initiates breast cancer invasion and metastasis. Shortly afterward, Tavazzoie and colleagues [9] of the Joan Massague group revealed that miR-335 suppresses metastasis and migration by targeting the transcription factor SOX4 and tenascin C, an extracellular matrix component. At the same time, Huang and coworkers [10] including the Reuven Agami group reported that miR-

miRNA = microRNA; PGC $=$ protein-coding gene. 
373 and miR-520c stimulate cancer cell migration and invasion, and proposed suppression of CD44 (which encodes a cell surface receptor for hyaluronan) to be the underlying mechanism. Taken together, these landmark studies reveal a fine balance of noncoding RNAs as stimulators and inhibitors of metastasis, and identify several targets that could potentially represent the molecular link between miRNA deregulation and a specific tumor behavior (Figure 1).

These are remarkable discoveries, not only because they open a new field of investigation but also because the authors finely dissected molecular pathways that are involved in breast tumor metastasis (Figure 1). The study by Ma and coworkers [8] revealed that upregulation of miR-10b promotes invasion and metastasis. Whereas miR-10b is downregulated in most breast cancers in comparison with normal breast tissue [11], this miRNA is over-expressed in about $50 \%$ of metastatic breast cancers [8]. The authors proved that ectopic expression of miR-10b had no effect on proliferation, but an increase in transwell migration and Matrigel invasion was acquired by cells of two different human breast cell lines. In vivo ectopic expression of miR-10b conferred invasive properties on otherwise noninvasive breast cancer cells; although control tumors could not invade surrounding tissues and exhibited poor vascularization, miR-10b over-expressing tumors exhibited an invasive behavior and were highly vascularized. Moreover, although lung micrometastases were detectable in miR-10b over-expressing cells, no intravasating cells or lung metastases were found in control tumors. Together, these findings indicate that miR-10b can promote metastasis in otherwise nonmetastatic breast cancer cells.

Then, Ma and coworkers [8] undertook the difficult task of dissecting the molecular pathway linking miR-10b to the metastatic process. First, they found that Twist, a metastasispromoting transcription factor, could induce miR-10b expression and demonstrated that miR-10b is an essential element in the Twist-induced metastasis program. Second, they proved that HOXD10, a homeobox transcription factor that promotes or maintains a differentiated phenotype in epithelial cells, is a target of miR-10b. HOXD10 is indeed expressed at low level in metastatic tumors. The authors demonstrated the importance of HOXD10 as an effector of miR-10b by showing that ectopic expression of HOXD10 that was unresponsive to miR-10b could abrogate miR-10b induced cell motility and invasiveness. Finally, the authors showed that RhoC, a G-protein involved in metastasis that is repressed by HOXD10, becomes strongly expressed in response to miR-10b expression. Importantly, reduction in RhoC expression by small interfering RNA caused repression of miR-10b induced cell migration and invasion, implying that $\mathrm{RhoC}$ is a downstream effector of miR-10b [8].

The work by Huang and coworkers [10] revealed that miR-373 and miR-520c are also metastasis-promoting micro-
Figure 1

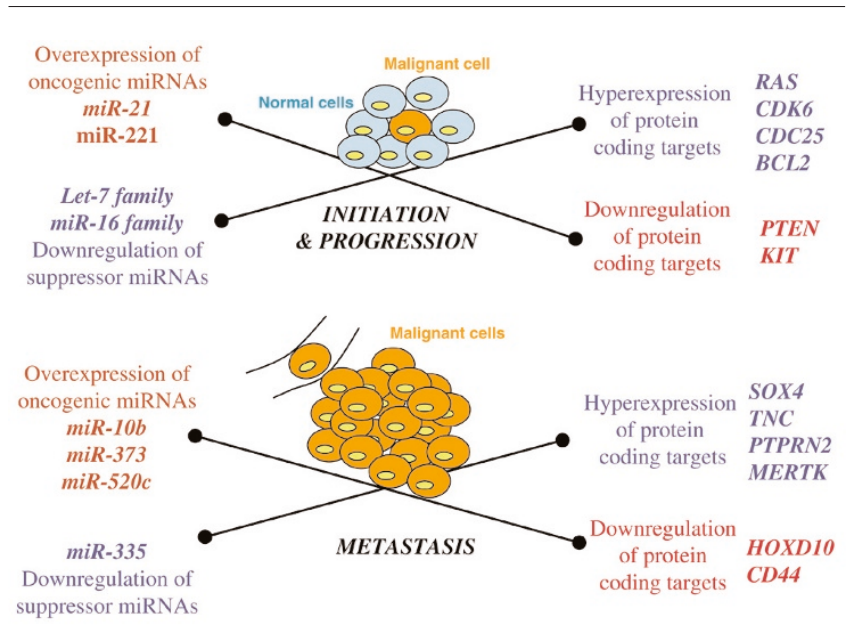

microRNAs and PCGs: involvement in early (initiation), intermediate (progression) and late (metastasis) steps in tumorigenesis. Presented are the specific instances in which let-7 and miR-16 families (already found to be involved in early stages of tumorigenesis), and miR-21 and $m i R-221$ proved to be involved in tumor progression. Also shown is the newly described balance between miR-10b, miR-373, miR-520c, and miR-335 in metastasis. The significant confirmed targets are also shown. miRNA, microRNA; PGC, protein-coding gene.

RNAs. miR-373 was previously found to be associated with testicular cancer but not with metastasis. Similarly to miR-10b, miR-373 and miR-520c did not affect cell proliferation, but promoted a migratory and invasive phenotype of MCF7 cells in vitro. Furthermore, MCF7 cells over-expressing miR-373 or miR-520c developed metastatic nodules, which were instead absent in control cells. Elucidating the molecular pathways involved, the authors found that miR-373 and miR-520c 'seed' sequences were similar, suggesting that they could regulate an overlapping set of gene targets. Among nine shared potential gene targets, CD44 (which encodes a cell surface receptor for hyaluronan) was found to be a direct target of both miR-373 and miR-520c. Most importantly, ectopic expression of an CD44 gene that was unresponsive to miR-373/miR-520c could reduce the migratory properties of MCF7 cells overexpressing miR-373/miR-520c. To verify the significance of these results in primary breast carcinomas, the authors analyzed 11 matched normal/tumor breast samples. They found that miR-373 was upregulated in cancer, in particular in tumors exhibiting lymph node metastasis. Moreover, an inverse correlation with CD44 expression was identified. These findings strengthen the significance of the studies performed in breast cancer cell lines.

In contrast to the other studies, the team led by Joan Massagué [9] found that miR-335, miR-126, and miR-206 are metastasis-suppressor miRNAs. To identify these miRNAs, they compared miRNA expression of the metastatic nodules versus the unselected breast cancer parental cells. These 
miRNAs were consistently downregulated in metastatic foci. Moreover, the authors found that restoring the expression of miR-335, miR-126, and miR-106 significantly decreased the number of metastatic foci. To verify the relevance of these findings in clinical samples, Massaguè and colleagues analyzed the expression of these miRNAs in primary tumors. They found that low expression of $m i R-335$ or $m i R-126$ was significantly associated with poor metastasis-free survival. Thus, these two miRNAs were markers for the likelihood of developing metastasis. To elucidate the molecular basis of these findings, the authors profiled metastatic cells with restored miR-335 expression. Six PGCs were suppressed by miR-335. Among these, knockdown of the transcription factor SOX4 and TNC (encoding tenascin C) diminished in vitro invasive ability and in vivo metastatic potential, indicating that these genes are critical effectors of metastasis, which are activated by loss of miR-335. Importantly, in an analysis of published datasets on breast tumor gene expression, the authors found that the expression of the six signature genes was significantly associated with a poor metastasis-free survival in a cohort of 368 patients. These findings suggest the potential use of $m i R-335$, its six PCG signature, and miR126 in prognostic stratification of breast cancer patients.

\section{The consequences for scientists and patients: a miRevolution in breast cancer}

As with all breakthrough reports, these studies - published over 4 months - have significant consequences. First, they demonstrate that combination genome-wide profiling followed by functional studies that involve over-expression and downregulation of miRNAs represent the approach that is most likely to yield advances in the field of noncoding RNA research. Abnormal expression of tumor miRNAs, characterized by differential levels of expression for mature and/or precursor miRNA sequences as compared with normal cells, has been proved to be the main abnormality, or 'miRNoma', in cancer cells. Ma and colleagues [8] found that although miR$10 \mathrm{~b}$ was expressed at low levels in samples from metastasisfree patients, this miRNA was overexpressed in about half of the metastasis positive patients, a result that reconciles the data with a previous published study in which miR-10b was found downregulated in primary breast tumours [11]. Thus, miR-10b was specifically over-expressed only in metastatic cells, offering a rationale to investigate this miRNA. Furthermore, Tavazoie and colleagues [9] found that expression of miR-335 (and miR-126) is lost in samples from patients with relapsed breast cancer and that this loss of expression is associated with poor distal metastases-free survival. Similarly, Huang and colleagues [10] identified significant upregulation of miR-373 in samples from patients with metastatic breast cancer. Functional and molecular studies were essential for elucidating the significance of the abnormal expression of this miRNA in breast tumorigenesis.

A second implication of the three studies is that both the main targets and the regulators of miRNA must be identified if our understanding of the role played by miRNAs in complex multistep processes (such as primary tumor invasion and metastasis) is to improve. In the specific case of miR-10b, this usually difficult process was rendered possible by the extended experience of the Whitehead Institute group in unraveling genetic programs that drive tumor metastasis. Weinberg's laboratory was involved in elucidating the Twist signaling pathway during normal development and the epithelial-mesenchymal transition program, and the implications of this pathway for tumor metastasis (for review [12]). The Twist/miR-10b/HOXD10/RHOC interrelation is certainly a first example of metastasis-related interplay between noncoding RNAs and cancer PCGs. In this regard, a recent study showed that miR-21 (a ubiquitously overexpressed miRNA in human cancers [13]) regulates cell migration and invasion in hepatocellular cancer [4]. Massague's group has a long track record of seminal discoveries related to the molecular basis of metastasis (for review [14]), whereas Agami's group has much experience in genetic screening with miRNA expression libraries, and previous elegant work by the latter [15] identified miR-373 as an oncogene in testicular germ cells tumors through direct inhibition of the expression of the tumor suppressor LATS2. The data reported by that group clearly demonstrate that the same miRNA could target distinct PCGs in different types of tumors and work in distinct signaling pathways.

Last but not at least, this recent work could have therapeutic implications for the future. Most deaths from cancer are associated with development of metastases, and prevention or elimination of disseminated disease would have a major impact on cancer mortality. The rationale for using miRNAs as potential therapeutic targets is underpinned by the fact that miRNA overexpression in cancer cells has a pathogenic effect [16]. Therefore, future phase I trials might demonstrate reduction in miR-10b, miR-373, and miR-520c levels in patients with advanced breast cancer by various agents such as antisense oligonucleotides against miRNAs, looked nucleic acids, antimiRNAs, or the antagomirs. On the other hand, over-expression of miR-335 by mimic miRNAs could be another option to be tested in patients. Although exciting, the use of miRNA-based gene therapy in metastatic human cancers must still demonstrate high efficiency of target inhibition, with significantly improved patient survival and minimal toxicity.

\section{Competing interests}

The authors declare that they have no competing interests.

\section{Acknowledgements}

Dr Negrini is supported by grants from the University of Ferrara and by project NOBEL from Fondazione Cariplo, Milan, Italy. Dr Calin is supported by the CLL Global Research Foundation and, in part, as a University of Texas System Regents Research Scholar and as a Fellow of The University of Texas MD Anderson Research Trust. We apologize to our colleagues whose work was not cited because of space limitations.

\section{References}

1. Calin GA, Dumitru CD, Shimizu M, Bichi R, Zupo S, Noch E, Aldler H, Rattan S, Keating M, Rai K, Rassenti L, Kipps T, Negrini $M$, Bullrich F, Croce CM: Frequent deletions and down-regula- 
tion of micro-RNA genes miR15 and miR16 at 13q14 in chronic lymphocytic leukemia. Proc Natl Acad Sci USA 2002, 99:15524-15529.

2. Vasudevan S, Tong $Y$, Steitz JA: Switching from repression to activation: MicroRNAs can up-regulate translation. Science 2007, 318:1931-1934.

3. Calin GA, Croce CM: MicroRNA-cancer connection: the beginning of a new tale. Cancer Res 2006, 66:7390-7394.

4. Meng F, Henson R, Wehbe-Janek H, Ghoshal K, Jacob ST, Patel T: MicroRNA-21 regulates expression of the PTEN tumor suppressor gene in human hepatocellular cancer. Gastroentero/ogy 2007, 133:647-658.

5. He X, He L, Hannon GJ: The guardian's little helper: microRNAs in the p53 tumor suppressor network. Cancer Res 2007, 67:11099-11101.

6. Chang TC, Yu D, Lee YS, Wentzel EA, Arking DE, West KM, Dang CV, Thomas-Tikhonenko A, Mendell JT: Widespread microRNA repression by Myc contributes to tumorigenesis. Nat Genet 2008, 40:43-50.

7. O'Donnell KA, Wentzel EA, Zeller KI, Dang CV, Mendell JT: cMyc-regulated microRNAs modulate E2F1 expression. Nature 2005, 435:839-843.

8. Ma L, Teruya-Feldstein J, Weinberg RA: Tumour invasion and metastasis initiated by microRNA-10b in breast cancer. Nature 2007, 449:682-688.

9. Tavazoie SF, Alarcon C, Oskarsson T, Padua D, Wang Q, Bos PD, Gerald WL, Massague J: Endogenous human microRNAs that suppress breast cancer metastasis. Nature 2008, 451: 147-152.

10. Huang Q, Gumireddy K, Schrier M, le Sage C, Nagel R, Nair S, Egan DA, Li A, Huang G, Klein-Szanto AJ, Gimotty PA, Katsaros D, Coukos G, Zhang L, Puré E, Agami R: The microRNAs miR373 and miR-520c promote tumour invasion and metastasis. Nat Cell Biol 2008, 10:292-210.

11. Iorio MV, Ferracin M, Liu CG, Veronese A, Spizzo R, Sabbioni S, Magri E, Pedriali M, Fabbri M, Campiglio M, Ménard S, Palazzo JP, Rosenberg A, Musiani P, Volinia S, Nenci I, Calin GA, Querzoli P, Negrini M, Croce CM: MicroRNA gene expression deregulation in human breast cancer. Cancer Res 2005, 65:7065-7070.

12. Yang J, Mani SA, Weinberg RA: Exploring a new twist on tumor metastasis. Cancer Res 2006, 66:4549-4552.

13. Volinia S, Calin GA, Liu CG, Ambs S, Cimmino A, Petrocca F, Visone $\mathrm{R}$, lorio $\mathrm{M}$, Roldo $\mathrm{C}$, Ferracin $\mathrm{M}$, Prueitt RL, Yanaihara $\mathrm{N}$, Lanza G, Scarpa A, Vecchione A, Negrini M, Harris CC, Croce CM: A microRNA expression signature of human solid tumors defines cancer gene targets. Proc Natl Acad Sci USA 2006, 103:2257-2261.

14. Nguyen DX, Massague J: Genetic determinants of cancer metastasis. Nat Rev 2007, 8:341-352.

15. Voorhoeve PM, le Sage C, Schrier M, Gillis AJ, Stoop H, Nagel R, Liu YP, van Duijse J, Drost J, Griekspoor A, Zlotorynski E, Yabuta N, De Vita G, Nojima H, Looijenga LH, Agami R: A genetic screen implicates miRNA-372 and miRNA-373 as oncogenes in testicular germ cell tumors. Cell 2006, 124:1169-1181.

16. Tili E, Michaille JJ, Gandhi V, Plunkett W, Sampath D, Calin GA: miRNAs and their potential for use against cancer and other diseases. Future Oncol 2007, 3:521-537. 